\title{
Qualidade nutricional e estabilidade oxidativa de manteigas produzidas do leite de vacas alimentadas com cana-de-açúcar suplementada com óleo de girassol
}

\author{
[Nutritional quality and oxidative stability of butter obtained from cows fed sugar-cane \\ supplemented with sunflower oil] \\ P.A.V. Barros ${ }^{1}$, M.B.A. Glória ${ }^{2,7}$, F.C.F. Lopes $^{3,7}$, M.A.S. Gama ${ }^{3}$, S.M. Souza ${ }^{4,7}$, \\ M.H.F Mourthe $e^{5,8}$, M.I. Leão ${ }^{6}$ \\ ${ }^{1}$ Aluna de pós-graduação - Escola de Veterinária - Universidade de Federal de Minas Gerais - Belo Horizonte, MG \\ ${ }^{2}$ Faculdade de Farmácia - Universidade de Federal de Minas Gerais - Belo Horizonte, MG \\ ${ }^{3}$ Embrapa Gado de Leite - Juiz de Fora, MG \\ ${ }^{4}$ Rowett Institute, University of Aberdeen, Scotland, UK \\ ${ }^{5}$ Universidade Federal dos Vales do Jequitinhonha e Mucuri - Diamantina, MG \\ ${ }^{6}$ Universidade Federal de Viçosa - Viçosa, MG \\ ${ }^{7}$ Bolsista do CNPq \\ ${ }^{8}$ Bolsista da Capes \\ RESUMO
}

\begin{abstract}
O objetivo deste estudo foi avaliar o perfil de ácidos graxos (AG), os índices de qualidade nutricional e a estabilidade oxidativa (EO) de manteigas produzidas do leite de vacas alimentadas com dietas à base de cana-de-açúcar contendo níveis crescentes de óleo de girassol (OG): 0 (controle); 1,5; 3,0 e 4,5\% da matéria seca (MS). O perfil de AG das manteigas foi analisado por cromatografia gasosa, e a EO foi determinada utilizando-se o equipamento Rancimat ${ }^{\circledR}$, modelo 743 , operado a $120^{\circ} \mathrm{C}$ e fluxo de ar de 20L/h. As concentrações dos AG rumênico (CLA cis-9, trans-11), vacênico (C18:1 trans-11) e oleico (C18:1 cis-9) na gordura das manteigas foram aumentadas em 867, 687 e 148\%, respectivamente, à medida que se aumentou de 0 para $4,5 \%$ o nível de OG na dieta. Por outro lado, as concentrações dos AG saturados de cadeia média foram linearmente reduzidas $(\mathrm{P}<0,0001)$ na gordura das manteigas, em razão do incremento de OG nas dietas. Quanto aos índices de qualidade nutricional, houve redução linear $(\mathrm{P}<0,0001)$ no índice de aterogenicidade e no de trombogenicidade e aumento da relação entre AG hipo e hipercolesterolêmicos, em resposta ao aumento do nível de OG na dieta. Consistente com o incremento $(\mathrm{P}<0,0001)$ nas concentrações totais dos AG mono e poli-insaturados, a EO da gordura das manteigas foi linearmente reduzida $(\mathrm{P}<0,0001)$ em razão do incremento de $\mathrm{OG}$ nas dietas. Concluiu-se que a suplementação com OG melhorou a qualidade nutricional das manteigas produzidas do leite de vacas Holandês x Gir devido a mudanças positivas no perfil de AG da gordura. Entretanto, tais mudanças na composição dos AG da gordura foram acompanhadas de redução da EO das manteigas, associada à menor vida de prateleira.
\end{abstract}

Palavras-chave: ácido graxo, CLA, produto lácteo, rancidez oxidativa, saúde humana

\begin{abstract}
The objective of this study was to evaluate the fatty acid (FA) profile, nutritional quality and oxidative stability $(O E)$ indexes of butter obtained from milk of cows fed sugar cane-based diets containing increasing levels of sunflower oil (SO): 0 (Control); 1.5; 3.0 and 4.5\% of diet DM. The butter FA profile was analyzed by gas chromatography and OE was determined using the Rancimat ${ }^{\circledR}$ equipment (model 743 ) operated at $120^{\circ} \mathrm{C}$ and air flow of $20 \mathrm{~L} / \mathrm{h}$. The concentrations of rumenic acid (cis-9 trans-11 CLA), vaccenic acid (trans-11 C18:1) and oleic acid (cis-9 C18:1) in butter fat were increased by 867, 687 and $148 \%$, respectively, as the dietary SO level increased from 0 to $4.5 \%$. In contrast, the concentrations of
\end{abstract}

Recebido em 13 de abril de 2012

Aceito em 15 de março de 2013

E-mail:pattyybarros@gmail.com 
medium chain saturated FA were linearly reduced $(P<0.05)$ in butter fat from cows fed increasing levels of SO. Regarding the butter nutritional quality, a linear decrease $(P<0.0001)$ in atherogenicity and thrombogenicity indexes and a linear increase $(P<0.0001)$ in the hypocholesterolemic/ hypercholesterolemic ratio were observed compared to control, 1.5, 3.0 and $4.5 \%$ SO, respectively. Consistent with the increased $(P<0.0001)$ concentration of mono and polyunsaturated $F A$, the $O E$ of butter fat was linearly reduced $(P<0.0001)$ as the dietary SO level increased. It was concluded that diet supplementation with SO improved the nutritional quality of butter fat of Holstein $x$ Gir dairy cows as a result of positive changes in milk FA profile. However, these changes in milk FA composition were accompanied by a reduction in the SO of butter, which in turn is associated with a shorter shelf life.

Keywords: fatty acid, CLA, oxidative rancidity, dairy product. human health

\section{INTRODUÇÃO}

Nos últimos anos, diversas pesquisas foram realizadas visando tornar a composição da gordura do leite mais adequada ao consumo humano (Lopes et al., 2008, 2009). Com foco na redução do risco de doenças cardiovasculares, tais pesquisas têm buscado a diminuição dos teores dos ácidos graxos (AG) saturados de cadeia média, como o láurico (C12:0), o mirístico (C14:0) e o palmítico (C16:0) - considerados aterogênicos e hipercolesterolêmicos, bem como o incremento da concentração do ácido oleico (C18:1 cis-9) no leite, considerado hipocolesterolêmico (Lopes et al., 2011a, 2011b). Outro objetivo dessas pesquisas tem sido elevar a concentração do ácido rumênico (CLA cis-9, trans-11), principal isômero de CLA (do inglês conjugated linoleic acids) presente no leite bovino, cujas propriedades anticarcinogênicas, antiaterogênicas, antidiabetogênicas (diabetes do tipo II) e imunomodulatórias têm sido demonstradas (Lopes et al., 2011b). O aumento da concentração do ácido vacênico (C18:1 trans11) nos produtos lácteos também é importante, pois foram encontrados $19 \%$ de conversão deste em ácido rumênico em humanos (Wijlen e Colombani, 2010). Além disso, o ácido vacênico é o principal precursor para a síntese endógena do ácido rumênico na glândula mamária (Bauman et al., 2000).

Existem vários índices que podem auxiliar e complementar estudos de avaliação do perfil de AG do leite e de derivados lácteos. Como exemplo, podem-se citar os índices de aterogenicidade e trombogenicidade (Ulbricht e Southgate, 1991), além das razões entre os AG hipocolesterolêmicos e hipercolesterolêmicos h/H (Santos - Silva et al., 2002) e entre os AG poli-insaturados (AGPI) $\omega-6 / \omega-3$ (Martin et al., 2006).

Um aspecto merecedor de mais estudos, por sua importância do ponto de vista tecnológico e de comercialização do leite e de produtos lácteos, diz respeito à estabilidade oxidativa (EO) de suas gorduras, diretamente relacionada à sua "vida de prateleira" (Lopes et al., 2011b). Redução linear na EO de manteigas obtidas do fornecimento às vacas de dietas suplementadas com níveis de óleo de soja foi relatada por Gama et al. (2008).

Não foi encontrado na literatura nenhum estudo de avaliação do perfil de AG e da qualidade nutricional e estabilidade oxidativa de manteigas produzidas do leite de vacas recebendo dietas suplementadas com óleos vegetais, baseadas em cana-de-açúcar, importante forrageira tropical utilizada na estação seca do ano no Brasil.

Este trabalho tem como objetivo avaliar o efeito da inclusão de níveis crescentes de óleo de girassol na dieta de vacas Holandês $x$ Gir recebendo cana-de-açúcar, sobre o perfil de AG, os índices de qualidade nutricional e a estabilidade oxidativa das manteigas.

\section{MATERIAL E METODOS}

O experimento foi realizado na Embrapa Gado de Leite (Coronel Pacheco, MG), sendo utilizadas 12 vacas Holandês x Gir, com média de produção de $18,2 \mathrm{~kg} /$ dia de leite $(3,1 \%$ de gordura; $2,9 \%$ de proteína; $4,3 \%$ de lactose e $11,3 \%$ de extrato seco total), em delineamento com três quadrados latinos (QL) 4x4. Foram avaliadas quatro dietas baseadas em $60 \%$ de cana-de-açúcar picada e $40 \%$ de concentrado, suplementadas com $0 ; 1,5 ; 3,0$ e 4,5\% de óleo de girassol (OG), com base na matéria seca (MS). As composições químicas médias (\% da MS) das 
quatro dietas foram, respectivamente: 12,$9 ; 12,8$; 12,$7 ; 12,6 \%$ de proteína bruta; 31,$1 ; 31,2 ; 31,5$ e $31,3 \%$ de fibra em detergente neutro corrigida para cinzas e proteína; e 1,$8 ; 3,2 ; 4,5$ e $5,9 \%$ de extrato etéreo. A cana-de-açúcar e o OG apresentaram, respectivamente, 12,7 e $22,1 \mathrm{~g}$ de ácido oleico/100g de AG totais; 30,7 e 59,9g de ácido linoleico/100g de AG totais; e 10,7 e 1,4g de ácido $\alpha$-linolênico/100g de AG totais.

As manteigas foram produzidas por processo semiartesanal (Gonzales et al., 2003), no primeiro dia de coleta de cada fase do QL, pois há relatos na literatura (Bauman et al., 2000) de que as maiores concentrações de CLA na gordura do leite são observadas de sete a 10 dias após o início do fornecimento de dietas ricas em óleo, sendo obtida uma manteiga por tratamento em cada fase do QL, totalizando 16 manteigas, que foram armazenadas a $-20^{\circ} \mathrm{C}$ (Gonzales et al., 2003) até a realização das análises de acidez, umidade e gordura (Brasil, 1996). Para determinação da cor, utilizou-se metodologia descrita por Georgantelis et al. (2007).

A determinação do perfil de ácidos graxos foi realizada no Laboratório de Cromatografia da Embrapa Gado de Leite (Juiz de Fora, MG). Utilizou-se cromatógrafo de fase gasosa, modelo 6890N (Agilent Technologies Inc., EUA), equipado com coluna capilar CP-SIL 88 (100m x $0,25 \mathrm{~mm} \times 0,2 \mu \mathrm{m}$; Varian Inc., EUA), e detector de ionização de chama. As condições cromatográficas e a programação de temperatura foram as descritas por Cruz-Hernandez et al. (2007).

A avaliação nutricional da gordura das manteigas foi realizada por meio dos índices de aterogenicidade $[\mathrm{IA}=[(\mathrm{C} 12: 0)+(4 \times \mathrm{C} 14: 0)+$ (C16:0)]/[(AGPI $\omega-6+$ AGPI $\omega-3)+$ AGMI) $]]$, de trombogenicidade $[\mathrm{IT}=(\mathrm{C} 14: 0+\mathrm{C} 16: 0+$ C18:0 $) /[(0,5 \times$ AGMI $)+(0,5 \times$ AGPI $n-6)+(3 \times$ AGPI $\omega-3)+($ AGPI $\omega$-3/AGPI $\omega-6)$ ]] (Ulbricht e Southgate, 1991); pela razão entre AG hipocolesterolêmicos e hipercolesterolêmicos $[\mathrm{h} / \mathrm{H}=\mathrm{C} 18: 1$ cis -9 + C18:1 trans-15 + AGPI $\omega-6$ + AGPI $\omega-3 / \mathrm{C} 12: 0$ + C14:0 + C16:0] (SantosSilva et al., 2002); e AGPI $\omega-6 / \omega-3=[(18: 2$ cis9 trans- $12+18: 2$ cis-9 cis-12+ 18:2 trans-10 cis-
12+ C20:0 cis-11 cis-14+ C20:3 $\omega-6+\mathrm{C} 20: 4 \omega-$ 6) $/(\mathrm{C} 18: 3 \omega-3$ isômeros $+\mathrm{C} 18: 3$ cis- $9-$ cis-12 cis-15)] (Martin et al., 2006), em que AGMI = AG monoinsaturados.

Utilizou-se método adaptado de Anwar et al. (2003) para determinação da estabilidade oxidativa (EO) das manteigas. As amostras foram aquecidas em estufa $\left(40^{\circ} \mathrm{C}\right)$ em tubos de vidro até a separação das fases líquida e oleosa, sendo, imediatamente após, filtradas em papel de filtro qualitativo, e foram pesados $5 \mathrm{~g}$ de matéria oleosa em tubos de ensaio. No aparelho Rancimat $^{\circledR}$, modelo 743 (Metrohm), as amostras de matéria oleosa, em duplicata, foram analisadas à temperatura de $120^{\circ} \mathrm{C}$, com fluxo de ar de $20 \mathrm{~L} / \mathrm{h}$ e $60 \mathrm{~mL}$ de água Milli-Q para recepção dos ácidos voláteis.

Os efeitos dos níveis de OG nas dietas sobre o perfil de AG, os índices de qualidade nutricional e a estabilidade oxidativa da gordura das manteigas do leite foram avaliados em delineamento QL 4 x 4, por meio de análises de regressão linear e quadrática $(\alpha=0,05)$ pelo PROC REG do SAS (Statistical..., 2002). Utilizou-se o LSMEANS do PROC GLM (Statistical..., 2002) para geração das médias das variáveis e respectivos erros-padrão. Os procedimentos experimentais utilizados seguiram os princípios de ética e bem-estar animal da Embrapa Gado de Leite.

\section{RESULTADOS E DISCUSSÕES}

A adição crescente de OG nas dietas das vacas promoveu alteração no perfil de AG das manteigas (Tab. 1). Observou-se que as concentrações dos AG de cadeia curta $(\leq \mathrm{C} 10: 0)$ e média (C12:0 a C16:0) foram linearmente reduzidas $(\mathrm{P}<0,05)$ em razão do incremento no nível de OG nas dietas. Já a maioria dos AG de cadeia longa $(\geq \mathrm{C} 18: 0)$ apresentaram aumento linear $(\mathrm{P}<0,05)$ em suas concentrações. $\mathrm{O}$ perfil de AG do OG e da cana-de-açúcar, com elevado teor do ácido linoleico $(59,9$ e 30,7\%, respectivamente), a bio-hidrogenação ruminal e o metabolismo na glândula mamária podem ser considerados os principais responsáveis pelas respostas encontradas. 


\section{Barros et al.}

Tabela 1. Concentrações dos principais ácidos graxos - AG (g/100g de AG totais) nas manteigas produzidas do leite de vacas alimentadas com cana-de-açúcar suplementada com níveis crescentes de óleo de girassol (OG) na matéria seca (MS) da dieta

\begin{tabular}{|c|c|c|c|c|c|c|c|}
\hline \multirow{2}{*}{$\begin{array}{l}\text { Ácido graxo (AG) } \\
\text { (g/100 g de AG totais) }^{1}\end{array}$} & \multicolumn{4}{|c|}{ Níveis de inclusão de OG (\% da MS) } & \multirow{2}{*}{$\mathrm{EPM}^{2}$} & \multirow{2}{*}{$\begin{array}{l}\text { Valor de } \\
\text { P }\end{array}$} & \multirow{2}{*}{$\begin{array}{l}\text { Equação de } \\
\text { regressão }\end{array}$} \\
\hline & 0 & 1,5 & 3,0 & 4,5 & & & \\
\hline $\mathrm{C} 4: 0$ & 3,5 & 3,7 & 3,3 & 2,8 & 0,1889 & 0,0028 & $\hat{\mathrm{y}}=3,8-0,17 \mathrm{x} ; \mathrm{r}^{2}=0,45$ \\
\hline C6:0 & 2,2 & 2,1 & 1,6 & 1,3 & 0,0819 & $<0,0001$ & $\hat{y}=2,3-0,20 x ; r^{2}=0,87$ \\
\hline C8:0 & 1,4 & 1,2 & 0,9 & 0,7 & 0,0420 & $<0,0001$ & $\hat{y}=1,42-0,15 x ; r^{2}=0,94$ \\
\hline C10:0 & 3,5 & 2,8 & 2,2 & 1,7 & 0,1048 & $<0,0001$ & $\hat{y}=3,5-0,41 x ; r^{2}=0,96$ \\
\hline $\mathrm{C} 12: 0$ & 4,3 & 3,3 & 2,4 & 1,9 & 0,1099 & $<0,0001$ & $\hat{y}=4,2-0,52 x ; r^{2}=0,89$ \\
\hline C14:0 & 12,5 & 11,2 & 9,2 & 8,2 & 0,2114 & $<0,0001$ & $\hat{y}=12,5-0,98 x ; r^{2}=0,92$ \\
\hline C16:0 & 36,4 & 29,3 & 24,1 & 22,1 & 0,8902 & $<0,0001$ & $\hat{y}=35,2-3,2 x ; r^{2}=0,86$ \\
\hline C18:0 & 7,1 & 11,0 & 12,6 & 12,5 & 0,5379 & $<0,0001$ & $\hat{y}=7,19+3,16 x-0,44 x^{2} ; r^{2}=0,83$ \\
\hline C18:1 trans -9 & 0,1 & 0,2 & 0,4 & 0,7 & 0,0223 & $<0,0001$ & $\hat{y}=0,11+0,12 x ; r^{2}=0,89$ \\
\hline C18:1 trans -10 & 0,2 & 0,6 & 1,2 & 3,1 & 0,5735 & $<0,0001$ & $\hat{y}=0,60 x ; r^{2}=0,69$ \\
\hline C18:1 trans -11 & 0,8 & 2,4 & 5,4 & 6,9 & 0,6327 & $<0,0001$ & $\hat{y}=0,60 x ; r^{2}=0,94$ \\
\hline C18:1 cis-9 + C18:1 trans- 15 & 13,6 & 17,1 & 19,5 & 20,1 & 0,5261 & $<0,0001$ & $\hat{y}=14,29+1,46 x ; r^{2}=0,85$ \\
\hline CLA cis -9 trans -12 & 0,08 & 0,08 & 0,07 & 0,06 & 0,0043 & $<0,0001$ & $\hat{y}=0,08-0,005 x ; r^{2}=0,74$ \\
\hline C18:2 cis-9 cis-12 & 1,80 & 1,90 & 2,04 & 1,90 & 0,0695 & $\mathrm{~ns}^{3}$ & $\mathrm{~ns}$ \\
\hline C20:0 & 0,11 & 0,14 & 0,14 & 0,13 & 0,0057 & $\mathrm{~ns}$ & $\hat{y}=0,11+0,02 x-0,003 x^{2} ; r^{2}=0,45$ \\
\hline C20:1 cis-11 & 0,04 & 0,04 & 0,06 & 0,06 & 0,0020 & $<0,0001$ & $\hat{y}=0,03+0,007 x ; r^{2}=0,93$ \\
\hline C18:3 cis-9 cis-12 cis- 15 & 0,14 & 0,13 & 0,13 & 0,12 & 0,0050 & 0,0004 & $\hat{y}=0,14-0,005 x ; r^{2}=0,58$ \\
\hline CLA cis-9 trans-11 & 0,38 & 1,02 & 2,06 & 2,61 & 0,2921 & $<0,0001$ & $\hat{y}=0,61 x ; r^{2}=0,92$ \\
\hline CLA trans-9 cis-11 & 0,02 & 0,04 & 0,08 & 0,12 & 0,0073 & $<0,0001$ & $\hat{y}=0,02 x ; r^{2}=0,95$ \\
\hline $\mathrm{C} 21: 0$ & 0,04 & 0,05 & 0,08 & 0,13 & 0,0109 & 0,0002 & $\hat{\mathrm{y}}=0,02+0,02 \mathrm{x} ; \mathrm{r}^{2}=0,61$ \\
\hline CLA trans -10 cis-12 & 0,01 & 0,02 & 0,03 & 0,03 & 0,0035 & $<0,0001$ & $\hat{y}=0,009+0,004 x ; r^{2}=0,68$ \\
\hline $\mathrm{C} 20: 2$ cis-11 cis-14 & 0,02 & 0,03 & 0,02 & 0,02 & 0,0032 & ns & $\mathrm{ns}$ \\
\hline $\mathrm{C} 22: 0$ & 0,04 & 0,06 & 0,06 & 0,06 & 0,0028 & 0,0075 & $\hat{y}=0,03+0,01 x-0,002 x^{2} ; r^{2}=0,75$ \\
\hline $\mathrm{C} 20: 3 \omega-6$ & 0,07 & 0,06 & 0,05 & 0,05 & 0,0047 & $<0,0001$ & $\hat{y}=0,07-0,005 x ; r^{2}=0,66$ \\
\hline $\mathrm{C} 20: 4 \omega-6$ & 0,14 & 0,12 & 0,10 & 0,08 & 0,0092 & $<0,0001$ & $\hat{y}=0,14-0,01 x ; r^{2}=0,74$ \\
\hline $\mathrm{C} 24: 0$ & 0,02 & 0,02 & 0,02 & 0,02 & 0,0024 & $\mathrm{~ns}$ & ns \\
\hline$\Sigma$ AG saturados cadeia curta & 10,7 & 10,0 & 8,28 & 6,65 & 0,3328 & $<0,0001$ & $\hat{y}=11,06-0,94 x ; r^{2}=0,91$ \\
\hline$\Sigma$ AG saturados cadeia média & 56,2 & 46,2 & 37,6 & 33,8 & 1,0494 & $<0,0001$ & $\hat{\mathrm{y}}=54,81-5,03 x ; \mathrm{r}^{2}=0,90$ \\
\hline$\Sigma$ AG saturados cadeia longa & 7,36 & 11,3 & 12,8 & 12,8 & 0,5444 & $<0,0001$ & $\hat{y}=7,40-3,19 x ; r^{2}=0,83$ \\
\hline$\sum$ AG monoinsaturados & 20,3 & 26,6 & 33,6 & 38,3 & 0,8059 & $<0,0001$ & $\hat{\mathrm{y}}=20,56+4,07 x ; \mathrm{r}^{2}=0,97$ \\
\hline$\sum$ AG poli-insaturados & 2,77 & 3,49 & 4,67 & 5,06 & 0,3550 & $<0,0001$ & $\hat{y}=2,79+0,53 x ; r^{2}=0,68$ \\
\hline
\end{tabular}

${ }^{1}$ Os AG estão ordenados de acordo com a ordem de eluição no cromatograma; ${ }^{2} \mathrm{EPM}=$ erro-padrão da média; ${ }^{3} \mathrm{~ns}=$ não significativo $(\mathrm{P}>0,05)$.

A redução linear $(\mathrm{P}<0,003)$ observada para a concentração do ácido butírico (C4:0) pode ser interpretada como de efeito negativo para a qualidade da manteiga, pois a este AG têm sido atribuídas propriedades antineoplásicas (Bauman et al., 2000). Da mesma forma, as concentrações de todos os AG saturados de cadeia curta também se reduziram com a adição crescente de OG, o que indica menor síntese de novo de AG na glândula mamária, já que estes são 
sintetizados do acetato e do $\beta$-hidroxibutirato, oriundos da corrente sanguínea (Grummer e Carroll, 1991).

As concentrações dos AG láurico (C12:0), mirístico (C14:0) e palmítico (C16:0) foram linearmente reduzidas $(\mathrm{P}<0,0001)$ com a adição crescente do OG. A redução foi de 53; 34 e 39\%, respectivamente, para as concentrações desses AG nas manteigas do tratamento sem inclusão de OG em relação às obtidas do leite das vacas que receberam dieta com 4,5\% de OG. Estes resultados podem favorecer o aumento do valor nutricional das manteigas produzidas das dietas acrescidas de OG e, consequentemente, propiciar benefícios para os consumidores, já que estes AG de cadeia média saturada são considerados aterogênicos (Tholstrup et al., 2004). Ribeiro (2009) também relatou redução dos teores dos AG láurico (51\%), mirístico (37\%) e palmítico $(69 \%)$ em manteigas oriundas do leite de vacas que receberam 0 e $4,5 \%$ de óleo de soja em dieta à base de capim-elefante (Pennisetum purpureum).

Houve efeito quadrático $(\mathrm{P}<0,0001)$ sobre a concentração do $\mathrm{AG}$ esteárico $(\mathrm{C} 18: 0)$ em razão da adição crescente de $O G$ na dieta. $O$ ácido esteárico é o produto final da bio-hidrogenação ruminal dos AGPI e AGMI com cadeia de $18 \mathrm{C}$ e, portanto, o aumento de sua concentração nas manteigas refletiu extensa bio-hidrogenação destes AG.

As concentrações dos AG oleico e vacênico aumentaram linearmente $(\mathrm{P}<0,0001)$ com a adição crescente de OG. O aumento observado para a concentração do ácido oleico é importante do ponto de vista nutricional da manteiga, pois a este AG são atribuídos efeitos anticolesterolêmicos (Tsimikas et al., 1999). Este resultado, provavelmente, foi devido à presença do ácido oleico na cana-de-açúcar, e, principalmente, no OG (respectivamente, $13 \mathrm{e}$ $22 \%$ dos AG totais), como também à sua síntese na glândula mamária, a partir do ácido esteárico (Jenkins et al., 2008).

Da mesma forma, o aumento do teor do ácido vacênico contribui para aumentar o valor nutricional da manteiga, pois trata-se do principal precursor do ácido rumênico (CLA cis9, trans-11) na glândula mamária dos ruminantes. $\mathrm{O}$ aumento da concentração do ácido vacênico advém da bio-hidrogenação ruminal dos AGPI, principalmente, do ácido linoleico, que é o AG com maior concentração no $\mathrm{OG}$ e também na cana-de-açúcar.

Foi observado aumento linear $(\mathrm{P}<0,05)$ da concentração do CLA cis-9, trans-11 nas manteigas em razão do incremento de $O G$ na dieta. Esse aumento pode ser explicado, principalmente, pela elevação da concentração do ácido vacênico. $\mathrm{O}$ tratamento com inclusão de 4,5\% de OG na dieta promoveu aumentos de 867 e $687 \%$, respectivamente, nas concentrações dos ácidos vacênico e rumênico em relação aos obtidos nas manteigas produzidas do leite das vacas que não receberam $O G$ na dieta. No trabalho de Lopes et al. (2009), os aumentos dos teores dos ácidos rumênico e vacênico foram de 359 e $467 \%$, respectivamente, nas manteigas produzidas do leite de vacas recebendo dietas com inclusão de $4,5 \%$ de óleo de soja em comparação às obtidas de dieta sem inclusão do óleo de soja. Essas respostas são consideradas benéficas devido às propriedades nutracêuticas atribuídas ao CLA cis-9, trans-11, como também ao potencial de produção deste em humanos a partir do ácido vacênico (Turpeinen et al., 2008). Outro aspecto relevante é a possibilidade de maior valorização nutricional da manteiga que, por seu elevado teor de AG, é associada apenas aos efeitos negativos que alguns destes possam causar à saúde humana.

Com relação ao ácido linoleico (C18:2 cis-9, cis-12), não houve efeito $(\mathrm{P}>0,05)$ das dietas sobre sua concentração, que foi, em média, de $1,91 \mathrm{~g} / 100 \mathrm{~g}$ de AG. Provavelmente, a bio-hidrogenação ruminal desse AG aumentou em razão da adição crescente de $\mathrm{OG}$ às dietas, de modo que a concentração final do ácido linoleico manteve-se semelhante. Corroborando isto, houve aumento linear $(\mathrm{P}<0,0001)$ das concentrações dos AG rumênico, vacênico e oleico, além de efeito quadrático $(\mathrm{P}<0,0001)$ na concentração de ácido esteárico (Tab. 1), todos estes AG originados, parcialmente, da biohidrogenação ruminal do ácido linoleico, principal AG do OG e da cana-de-açúcar.

A concentração do ácido $\alpha$-linolênico (C18:3 cis-9, cis-12, cis-15) foi linearmente reduzida ( $\mathrm{P}$ $=0,0004)$ nas manteigas com a adição crescente de OG na dieta das vacas, provavelmente, em razão da extensa bio-hidrogenação desse $A G$ no 
rúmen (Lopes et al., 2011b). Diferentemente do ácido linoleico, a concentração do ácido $\alpha$-linolênico é pequena no OG e na cana-deaçúcar (respectivamente, 1,4 e 10,7\%), bem como nos demais ingredientes componentes da mistura concentrada. A concentração dos AGMI C18:1 trans-9 (ácido elaídico) e trans-10 apresentou aumento linear $(\mathrm{P}<0,0001)$ com a adição crescente de $\mathrm{OG}$ à dieta. Esses efeitos podem ser considerados negativos, haja vista as propriedades cancerígenas atribuídas ao ácido elaídico e ao C18:1 trans-10, por ser precursor do C18:2 trans-10, cis-12, principal responsável pela depressão do teor de gordura do leite em vacas (Piperova et al., 2000).
Os índices de aterogenicidade (IA) e de trombogenicidade (IT) das manteigas apresentaram redução linear $(\mathrm{P}<0,0001)$ com a adição crescente de OG nas dietas (Tab. 2). A redução dos valores desses índices é importante para toda a cadeia de lácteos, pois ocorreu tanto pelo decréscimo das concentrações dos AG láurico, mirístico e palmítico (aterogênicos e trombogênicos) quanto pelo aumento dos AGMI e AGPI, principalmente, do CLA cis-9, trans-11, considerados benéficos à saúde humana (Lopes et al., 2011b).

Tabela 2. Índices de aterogenicidade e trombogenicidade, relação de ácidos graxos (AG) hipocolesterolêmicos:hipercolesterolêmico (h/H), de AG poli-insaturados (AGPI) $\omega-6 / \omega$-3, e estabilidade oxidativa (EO) em manteigas produzidas do leite de vacas recebendo $0 ; 1,5 ; 3,0$ ou 4,5\% de óleo de girassol (OG) na matéria seca (MS) da dieta

\begin{tabular}{|c|c|c|c|c|c|c|c|}
\hline \multirow{2}{*}{ Índices } & \multicolumn{4}{|c|}{ Níveis de inclusão de OG (\% da MS) } & \multirow{2}{*}{$\mathrm{EPM}^{1}$} & \multirow{2}{*}{ Valor de P } & \multirow{2}{*}{ Equação de regressão } \\
\hline & 0 & 1,5 & 3,0 & 4,5 & & & \\
\hline Aterogenicidade & 4,02 & 2,70 & 1,80 & 1,41 & 0,1441 & $<0,0001$ & $\hat{y}=3,98-0,58 x ; r^{2}=0,87$ \\
\hline Trombogenicidade & 4,68 & 3,43 & 2,50 & 2,05 & 0,1732 & $<0,0001$ & $\hat{y}=4,49-0,598 x ; r^{2}=0,91$ \\
\hline $\mathrm{h} / \mathrm{H}$ & 0,30 & 0,45 & 0,62 & 0,70 & 0,0264 & $<0,0001$ & $\hat{y}=0,31-0,09 x ; r^{2}=0,87$ \\
\hline AGPI $\omega-6 / \omega-3$ & 9,38 & 10,00 & 10,63 & 10,70 & 0,1996 & $<0,0001$ & $\hat{y}=9,49+0,3 x ; r^{2}=0,74$ \\
\hline EA (horas) & 4,00 & 3,03 & 2,42 & 1,68 & 0,2622 & $<0,0001$ & $\hat{y}=3,91-0,50 x ; r^{2}=0,86$ \\
\hline
\end{tabular}

${ }^{1} \mathrm{EPM}=$ Erro-padrão da média.

Segundo Tonial et al. (2010), os IA e IT indicam o potencial de estímulo à agregação plaquetária, ou seja, quanto menores os valores de IA e IT, maior é a quantidade de AG antiaterogênicos presentes em determinado óleo/gordura e, consequentemente, maior é o potencial de prevenção ao aparecimento de doenças coronarianas. Desta forma, a redução desses índices demonstra o potencial que existe para a produção de manteigas com qualidade nutricional mais adequada para a saúde humana, pois, comumente, esse produto é referido apenas por seu teor de gordura total.

Os resultados corroboram os de Lopes et al. (2008) e Ribeiro et al. (2011a), que também observaram redução linear $(\mathrm{P}<0,05)$ no IA e no IT do leite de vacas de raças leiteiras recebendo capim-elefante picado e concentrados contendo níveis crescentes de óleo de soja e OG, respectivamente. No presente estudo, os IA e IT foram reduzidos em 65 e $56 \%$, respectivamente, quando comparadas as manteigas obtidas das dietas sem inclusão ou com 4,5\% de OG, sendo essas reduções maiores que as relatadas por Lopes et al. (2008), de 37 e $24 \%$, e por Ribeiro et al. (2011a), de 46 e 27\%, quando as dietas sem inclusão ou com $4,5 \%$ de óleo vegetal foram comparadas.

A relação $\mathrm{h} / \mathrm{H}$ das manteigas aumentou linearmente $(\mathrm{P}<0,0001)$ com a adição crescente de OG. De acordo com Bentes et al. (2009), quanto maior $\mathrm{h} / \mathrm{H}$, mais adequado nutricionalmente é o óleo ou a gordura dos alimentos. No presente estudo, houve aumento de $233 \%$ nessa relação quando comparadas as manteigas produzidas do leite das vacas que receberam dieta sem inclusão ou com $4,5 \%$ de OG. Portanto, esses resultados indicam que a adição crescente de $\mathrm{OG}$ tornou o perfil de $\mathrm{AG}$ das manteigas mais adequado para o consumo humano, com maior potencial para prevenir o aumento do colesterol sérico, principalmente da 
fração LDL e, consequentemente, para reduzir o risco de doenças cardiovasculares (Ascherio e Willette, 1995).

A relação dos AGPI $\omega-6 / \omega-3$ apresentou aumento linear $(\mathrm{P}<0,0001)$ com a adição crescente de OG nas dietas. Houve incremento de $12,3 \%$ nesse índice quando comparadas as manteigas dos tratamentos sem inclusão ou com $4,5 \%$ de OG na dieta. Segundo a FAO (1994), valores abaixo de 10,0 sugerem quantidades desejáveis à dieta para a prevenção de riscos cardiovasculares. Foi observado que os índices calculados das manteigas referentes às dietas com 1,$5 ; 3,0$ e $4,5 \%$ de OG foram superiores a 10 , e isto se deveu tanto à redução linear do ácido $\alpha$-linolênico como à ausência de efeito do ácido linoleico, que são, respectivamente, os principais AG $\omega-6$ e $\omega-3$.

Porém, deve ser considerado que a manteiga apenas compõe parte da dieta diária das pessoas e pode contribuir como importante fonte para atender os requisitos de AG essenciais, além de outros nutrientes também relevantes na dieta.

Apesar da melhoria no perfil dos AG e nos índices de qualidade nutricional da manteiga, a inclusão crescente de $\mathrm{OG}$ na dieta promoveu redução linear $(\mathrm{P}<0,0001)$ da EO das manteigas. Essa redução ocorreu devido ao aumento das concentrações dos AGMI (EO = 6,50305$0,12507 *$ AGMI; $\left.\mathrm{r}^{2}=0,64 ; \mathrm{P}=0,0001\right)$ e AGPI totais $\left(\mathrm{EO}=6,19561-0,85350 * \mathrm{AGPI} ; \mathrm{r}^{2}=0,66\right.$; $\mathrm{P}<0,0001)$, e concomitante decréscimo na concentração dos AG saturados totais - AGS $\left(\mathrm{EO}=-3,96627+10,625^{*} \mathrm{AGS} ; \mathrm{r}^{2}=0,65 ;\right.$ $\mathrm{P}<0,0001)$, fatores estes que contribuem para tornar os alimentos mais susceptíveis à oxidação (Gama et al., 2008; Ribeiro et al., 2011b). A redução de 4,0 para 1,68 h na EO das manteigas obtidas das dietas sem inclusão ou com 4,5\% de OG, respectivamente, pode ser indicativo do menor tempo de validade ("vida de prateleira"), o que pode ser indesejável para a indústria. Porém, essa análise não pode ser feita isoladamente, pois devem ser considerados os benefícios do aumento das concentrações dos ácidos vacênico e rumênico, concomitante à redução das concentrações dos AG láurico, mirístico e palmítico (Tab. 1).

A utilização dos aditivos vitamina E e selênio em dietas de vacas leiteiras suplementadas com OG aumentou a EO do leite quando este foi comparado ao de vacas que não receberam tais agentes antioxidantes (Saran Netto et al., 2011). Estas respostas podem ajudar a viabilizar a produção leite e derivados com elevados teores de AGPI benéficos à saúde humana, sem que haja perdas nutricionais e sensoriais durante seu armazenamento. Tais resultados corroboram os relatados por Gama et al. (2008) e Ribeiro et al. (2011b), que utilizaram condições experimentais semelhantes ao do presente estudo. EO de 2,69; 2,28; 1,74; 1,34 h (Gama et al., 2008) e de 6,89; 6,26; 5,70; 5,51 h (Ribeiro et al., 2011b) foram relatadas pelos autores em dietas à base de capim-elefante suplementadas com 0; 1,5; 3,0 e 4,5\% (base MS) de óleo de soja e de OG, respectivamente. Os autores também atribuíram estas respostas ao aumento dos teores de AGMI e AGPI com a adição crescente do óleo vegetal às dietas.

O aumento da concentração de AGMI e AGPI reduz o ponto de fusão da gordura do leite, gerando uma manteiga de consistência mais mole a uma dada temperatura (Gonzalez et al., 2003), o que, por sua vez, pode melhorar a aceitação do consumidor por esse produto lácteo.

A adição de OG na dieta das vacas provocou aumento linear $(\mathrm{P}<0,05)$ da acidez das manteigas, sendo que os valores observados variaram de 0,52 a $0,66 \mathrm{mmol} / 100 \mathrm{~g}$ para as manteigas obtidas das dietas sem inclusão ou com 4,5\% de OG. Tais valores estão dentro dos exigidos para manteigas, cujo valor máximo é de 3,0mmol/100g (Brasil, 1996). O teor de umidade das manteigas não foi influenciado pelos tratamentos $(\mathrm{P}>0,05)$ e variaram de 18,33 a $19,48 \%$; todas as manteigas analisadas apresentaram valores acima do máximo de $16 \%$ exigido no Brasil (1996). Este aspecto é positivo, demonstrando que, apesar de o processo de produção da manteiga ter sido semiartesanal, o produto obtido foi similar em termos de umidade. Os teores de gordura variaram de 85,9 a $83,5 \%$, não sendo observado efeito dos tratamentos sobre essa variável $(\mathrm{P}>0,05)$. Desta forma, todas as manteigas analisadas apresentaram valores acima do mínimo de $82 \%$ exigido no Brasil (1996). A cor da manteiga está associada ao teor de gordura e, portanto, passível de variações sazonais. A manteiga tem como característica cor levemente amarelada (Brasil, 1996). Os valores obtidos para as variáveis a* e 
$\mathrm{b}^{*}$ (intensidades de verde e de amarelo, respectivamente) não foram afetados pela adição de OG na dieta $(\mathrm{P}>0,05)$. Porém, a variável $\mathrm{L}^{*}$ (luminosidade da amostra) foi aumentada em razão da adição de OG na dieta $\left(\hat{y}=8.066,8+186,0 \mathrm{x} \mathrm{r}^{2}=0,19 ; \mathrm{P}<0,05\right)$.

\section{CONCLUSÕES}

Manteiga nutricionalmente mais adequada ao consumo humano, com menor concentração de ácidos graxos saturados de cadeia média e teores mais elevados dos ácidos oleico, vacênico e rumênico, pode ser produzida pela inclusão de óleo de girassol na dieta de vacas Holandês x Gir alimentadas com cana-de-açúcar. Entretanto, tais mudanças na composição dos ácidos graxos da gordura foram acompanhadas de redução da estabilidade oxidativa das manteigas, associada à menor vida de prateleira.

\section{AGRADECIMENTOS}

À Fapemig e ao CNPq, pelo financiamento do trabalho e pela concessão de bolsas.

\section{REFERÊNCIAS}

ANWAR, F.; BHANGER, M.I.; KAZI, T.G. Relationship between rancimat and active oxygen method values at varying temperatures for several oils and fats. JAOCS, v.80, p.151-155, 2003.

ASCHERIO, A.; WILLETTE, W.C. New directions in dietary studies of coronary heart Disease. J. Nutr., v.125.p.647S-655S, 1995.

BAUMAN, D.E.; BARBANO, D.M.; DWYER, D.A. et al. Production of butter with enhanced conjugated linoleic acid for use in biomedical studies with animal models. J. Dairy Sci., v.83, p.2422-2425, 2000.

BENTES, A.S.; SOUZA, H.A.L; MENDONÇA, X.M.F. et al. Caracterização física e química e perfil lipídico de três espécies de peixes amazônicos. Rev. Bras. Tecnol. Agroind., v.3, p.97-108, 2009.

BRASIL. Ministério da Agricultura e do Abastecimento. Resolução Portaria N ${ }^{\circ} 146$ de 07 de março de 1996.

CRUZ-HERNANDEZ, C.; KRAMER, J.K.G.; KENNELLY, J.J. et al. Evaluating the conjugated linoleic acid and trans 18:1 isomers in milk fat of dairy cows fed increasing amounts of sunflower oil and a constant level of fish oil. J. Dairy Sci., v.90, p.3786$3801,2007$.
FAO - FOOD AND AGRICULTURE ORGANIZATION. Fats and Oils in Human Nutrition. Roma: FAO, 1994. p.3621-3627.

GAMA, M.A.S.; LOPES, F.C.F.; RIGUEIRA, J.C.S. et al. Perfil de ácidos grasos y estabilidad oxidativa de mantecas elaboradas con leche de vacas que reciben dietas con aceite de soja. Tecnol. Láctea Latinoam., v.54, p.56-57, 2008.

GEORGANTELIS, D.; BLEKAS, G.; KATIKOU, P. et al. Effect of rosemary extract, chitosan and atocopherol on lipid oxidation and colour stability during frozen storage of beef burgers. Meat Science, v.75, p.256-264, 2007.

GONZALES, S.; DUNCAN, S.E.; O'KEEFE, S.F. et al. Oxidation and textural characteristics of butter and ice cream with modified fatty acid profiles. J. Dairy Sci., v.86, p.70-77, 2003.

GRUMMER, R.R.; CARROLL, D.J. Effects of dietary fat on metabolic disorders and reproductive performance of dairy cattle. J. Anim. Sci., v.68, p.3838-3852, 1991.

JENKINS, T.C.; WALLACE, R.J.; MOATE, P.J. et al. Recent advances in biohydrogenation of unsaturated fatty acids within the rumen microbial ecosystem. J. Anim. Sci., v.86, p.397, 2008.

LOPES, F.C.F.; BARROS, P.A.V.; BRUSCHI, J.H. et $a l$. Perfil de ácidos graxos no leite de vacas Holandês em pastagens tropicais suplementadas com dois níveis de concentrado. Arq. Bras. Med. Vet. Zootec., v.63, p.518-521, $2011 \mathrm{a}$.

LOPES, F.C.F.; GAMA, M.A.S.; RIBEIRO, C.G.S. et al. Produção de leite com alto teor de CLA Experiência brasileira. In: PEREIRA, L.G.R.; NOBRE, M.M. et al. (Org.). Pesquisa, desenvolvimento e inovação para sustentabilidade da bovinocultura leiteira. Juiz de Fora, MG: Embrapa Gado de Leite, p.251-296, 2011b.

LOPES, F.C.F.; RIBEIRO, C.G.S.; RIBEIRO, M.T. et al. Milk fatty acid profile from dairy cows fed increasing levels of soybean oil in diets based on tropical forage. In: INTERNATIONAL SYMPOSIUM ON RUMINANT PHYSIOLOGY, 11., 2009, Clermont-Ferrand. Proceedings... Clermont-Ferrand: INRA, 2009, p.588-589.

LOPES, F.C.F.; GAMA, M.A.S.; ANTONIASSI, R. et al. Índices de aterogenicidade e trombogenicidade de manteigas oriundas do leite de vacas consumindo dietas com óleo de soja. In: CONGRESSO BRASILEIRO DE CIÊNCIA E TECNOLOGIA DE ALIMENTOS, 21., 2008, Belo Horizonte. Anais... Belo Horizonte: SBCTA/UFMG, 2008. 
MARTIN, C.A; ALMEIDA, V.V.; RUIZ, M.R. et al. Ácidos graxos poliinsaturados ômega-3 e ômega-6: importância e ocorrência em alimentos. Rev. de Nutrição, v.19, p.761-770, 2006.

PIPEROVA, L.S.; TETER, B.B.; BRUCKENTAL, I. et al. Mammary lipogenic enzyme activity, trans fatty acids and conjugated linoleic acids are altered in lactating dairy cows fed a milk fat - depressing diet. $J$. Nut., v.130, p.2568-2574, 2000.

RIBEIRO, C.G.S. Niveis crescentes de óleo de soja em dietas à base de capim-elefante (Pennisetum purpureum, Schummack) picado para vacas mestiças. Parâmetros da fermentação e cinética ruminal, composição e perfil de ácidos graxos do leite. 2009. 118f. Dissertação (Mestrado em Zootecnia) - Escola de Veterinária da Universidade Federal de Minas Gerais, Belo Horizonte, 2009.

RIBEIRO, C.G.S.; GAMA, M.A.S.; STUMPF, M.T. et al. Índices de aterogenicidade e trombogenicidade da gordura do leite de vacas consumindo dietas à base de capim-elefante contendo níveis crescentes de óleo de girassol. In: REUNIÃO ANUAL DA SOCIEDADE BRASILEIRA DE ZOOTECNIA, 48., 2011, Belém. Anais... Belém: SBZ, 2011a.

RIBEIRO, C.G.S.; GAMA, M.A.S.; TEIXEIRA, M.R. et al. Estabilidade oxidativa da gordura do leite de vacas consumindo dietas à base de capim-elefante contendo níveis crescentes de óleo de girassol. In: REUNIÃO ANUAL DA SOCIEDADE BRASILEIRA DE ZOOTECNIA, 48., 2011, Belém. Anais... Belém: SBZ, 2011b.

SANTOS-SILVA, J.; BESSA, R.J.B.; SANTOSSILVA, F. Effect of genotype, feeding system and slaughter weight on the quality of light lambs: fatty and composition of meat. Liv. Prod. Sci., v.77, p.187194, 2002.
SARAN NETTO, A.; ZANETTI, M.A.; DELLAQUA, G.F. et al. Estabilidade oxidativa do leite de vacas em função da suplementação na dieta com óleo de girassol selênio e vitamina E. In: REUNIÃO ANUAL DA SOCIEDADE BRASILEIRA DE ZOOTECNIA, 48., 2011, Belém. Anais... Belém: SBZ, 2011.

STATISTICAL Analysis Sistem-SAS Institute Inc. SAS ${ }^{\circledR}$ User's Guide: Statistics, Version 5 Edition. Cary, NC: SAS Institute Inc., 2002.

THOLSTRUP, T.; EHNHOLM, C.; JAUHIAINEN, M. et al. Effects of medium-chain fatty acids and oleic acid on blood lipids, lipoproteins, glucose, insulin, and lipid transfer protein activities. Amer. J. Clin. Nutr., v.79, p.564-569, 2004.

TONIAL, I.B.; OLIVEIRA, D.F.; BRAVO, C.E.C. $e t$ al. Caracterização físico-química e perfil lipídico do salmão (Salmo salar L.). Alim. e Nutrição, v.21, p.9398, 2010.

TSIMIKAS, S.; PHILIS-TSIMIKAS, A.; ALEXOPOULOS, S. et al. LDL isolated from Greek subjects on a typical diet or from American subjects on an oleate-supplemented diet induces less monocyte chemotaxis and adhesion when exposed to oxidative stress. Arterioscl. Thrombos, and Vasc. Biol., v.19, p.122-130, 1999.

TURPEINEN, A.A.; YLONEN, N.; VON WILLEBRAND, E. et al. Immunological and metabolic effects of cis-9, trans-11-conjugated linoleic acid in subjects with birch pollen allergy. Brit. J. Nutr., v.100, p.112-119, 2008.

ULBRICHT, T.L.V; SOUTHGATE, D.A.T. Coronary heart disease: Seven dietary factors. Lancet, v.338, p.985-992, 1991.

WIJLEN, R.P.J.V.; COLOMBANI P.C. Grass-based ruminant production methods and human bioconversion of vaccenic acid with estimations of maximal dietary intake of conjugated linoleic acids. Int. Dairy J., v.20, p.433-448, 2010. 